\title{
Bruno Frère
}

\section{Max Scheler et la phénoménologie française}

"Le savoir affectif n'est pas un savoir intellectuel non encore explicité mais un savoir autre. Savoir affectivement est savoir autrement" 1

\footnotetext{
1. F. Alquié, La Conscience affective, Paris, Vrin, 1979, p. 173.
} 


\section{§1. Introduction : pour une lecture phénoménologique de la pensée de Max Scheler}

"Tout sentiment est sensible", déclarait la Critique de la Raison Pratique (1985a, [V, 75]). Rangé parmi "les principes pratiques déterminants matériels", il s'esquisse chaque fois de manière strictement subjective et empirique dans des "inclinations" toujours singulières et contingentes ([V, 41]). Comme le rappelle F. Alquié, la morale kantienne ne peut s'actualiser qu'à la condition de suspendre la modalité affective de notre rapport au monde. Le sentiment constituant cette "inopportunité violente" qui risque continuellement de pervertir notre orientation volontaire vers l'universalité de la loi morale, il faut s'en abstraire pour pouvoir légiférer. Affranchie du sentiment, la pensée moderne à partir de Kant peut enfin assurer que nos états affectifs n'ont pas "d'influence sur une résolution pour laquelle nous devons désormais employer notre raison" ([V, 161]).

Largement kantienne, la philosophie morale allemande de la fin du XIXe Siècle ne s'écartera guère de cette posture. Mais à l'aube de la phénoménologie et du XXe Siècle, Max Scheler s'emploiera à démontrer dans son ouvrage principal (Le Formalisme en éthique), que ce qui est justement dommageable dans la morale élaborée par l'auteur des trois Critiques, c'est qu'il reste fâcheusement redevable de la distinction hiérarchique entre sensibilité et rationalité ${ }^{2}$. L'ampleur que Kant a donnée à sa démarche rationaliste l'a amené à ignorer la pleine autonomie du sentiment et, par conséquent, à participer "au discrédit qui pèse traditionnellement sur elle" (M. Henry, 1963, p. 715). L'ambition de l'œuvre schelerienne est de mettre un terme définitif à ce discrédit car il résulte selon lui d'une confusion qui inverse l'ordre des priorités philosophiques. Kant part d'un présupposé où matérial ${ }^{3}$ et sensoriel se confondent à l'origine du sentiment, lequel s'en trouve relégué au second rang de l'analyse philosophique. Estimer que la raison régit seule la sphère de la moralité pure en tant qu' a priori, c'est ipso facto ignorer les sollicitations de la perception affective. C'est mettre prématurément un point final au questionnement éthique et moral sans avoir remarqué le rôle que les constituants originaires de l'esprit émotionnel, véritable a priori, y jouent. Scheler entend bien libérer l'a priori de cette théorie de "l'entendement informateur et législateur de la volonté rationnelle" où Kant le retenait enfermé (Scheler, 1955, p. 91). Il va s'agir de se l'octroyer non plus dans un jugement, mais dans les actes intentionnels de la perception affective qui nous ouvrent au monde authentique des valeurs morales. En somme, c'est à la fois la priorité et l'a priorité qu'il va falloir rendre à la sphère sentimentale et affective dans laquelle apparaissent ces valeurs.

Or aujourd'hui, ce constat indiquant combien Scheler joue l'affectivité contre l'intellectualisme moral de Kant est lui-même devenu tellement notoire que le débat se rapproche de l'épuisement. Il suffit d'ailleurs de considérer l'état actuel des recherches portant sur la philosophie schelerienne pour remarquer que la critique de la pensée rationnelle et ses réflexions personnalistes sur les divers

\footnotetext{
2. Le Formalisme en éthique et l'éthique matériale des valeurs, trad. M. de Gandillac, Paris, Gallimard, 1955.

${ }^{3}$. Nous conserverons de l'allemand le terme matérial comme M. de Gandillac. Nous aurions pu, avec les traducteurs de Husserl, employer simplement la notion de matériel, ce qui dans le contexte phénoménologique qui est le sien n'aurait en rien trahi Scheler, puisque, comme nous le verrons, pas plus que la matière d'une intuition (les valeurs) ne renvoie à quelque réalité sensible, la perception affective de celle-ci n'enveloppe une quelconque réalité externe. Cependant, le néologisme de Gandillac s'avère un atout précieux. Il nous permet d'éviter en effet dans notre lecture du Formalisme le risque de l'amalgame pernicieux entre une valeur matériale perçue affectivement et son support perçu par la sensibilité qui peut être matériel ( $c f r$. Infra §2). Nous dirons que tout matériel est matérial, mais que tout matérial n'est pas matériel car une valeur peut exister indépendamment d'un support.
} 
sentiments humains ont déjà fait l'objet de travaux spécialisés les plus pointus. Pour présenter son parti pris moral contre la toute puissante "raison raisonnante", les commentaires posent généralement les problèmes à partir de la juxtaposition que Scheler opère entre "expérience morale et expérience émotive" (G. Gurvitch, 1961, p. 101). En partant du principe que, dans le Formalisme, les visées intentionnelles "ne portent jamais que sur des qualités axiologiques d'ordre moral existant par soi", ces mêmes problèmes sont alors clos par un consensus sur sa pensée : étant donné que l'expérience émotive découvre (et s'identifie à) l'existence d'une expérience morale originale, la philosophie première pour Scheler consisterait d'abord en une "herméneutique morale" et éthique qui chercherait à déterminer les valeurs qui en composent la matière axiologique (M. Scheler, 1955, p. 196) ${ }^{4}$. Ensuite, elle chercherait à définir les différentes voies intentionnelles par lesquelles elles affectent chaque individu originairement en en faisant une personne d'abord douée d'émotion plutôt que de conscience réflexive ${ }^{5}$. La personne affectée prendrait le pas sur le législateur kantien.

Le but premier de cet article serait de s'installer dans un mode d'investigation moins fréquenté de ces mêmes problèmes et de revoir autrement l'espace fondateur de la démarche de Scheler dans le Formalisme. Il semble en effet qu'à force de se concentrer sur ses résultats obtenus contre Kant, on en est arrivé à réduire son système à des considérations éthiques. La plupart du temps les auteurs traitant des réserves que Scheler manifeste à l'égard de la philosophie kantienne engagent leurs analyses à partir de sa tendance morale personnaliste et envisagent simplement son projet phénoménologique comme un des moyens mobilisés pour la formaliser. Pourtant, il se peut que ce projet vaille pour lui-même et qu'il engendre la pensée schelerienne au moins tout autant qu'il ne l'accompagne. Il est temps de déplacer la réflexion et renouveler le questionnement par une nouvelle hypothèse : il apparaît qu'à la suite du Formalisme en éthique de 1912, c'est aussi et surtout par son usage de la méthode forgée par (et avec) Husserl qu'il parviendra à donner toute sa décisive portée à son refus du modèle kantien. L'expérience que voudraient tenter ces quelques pages serait une lecture de l'histoire de l'a priori schelerien et de son matérialisme axiologique non plus en termes personnalistes mais par le truchement de son usage tout particulier d'une démarche phénoménologique qu'il a contribué à ouvrir. Sans l'outillage conceptuel qu'il articula grâce à Husserl, l'enjeu de ses critiques du formalisme et du rationalisme kantien n'aurait pu revêtir la pertinence qui fut la leur (Frère, 2004) ${ }^{6}$. Il est peut-être temps de parier sur le Scheler phénoménologue si l'on désire progresser dans les recherches philosophiques axées sur l'affectivité ou l'apriorité. La question devient alors en fin de parcours : jusqu'où ce pari peut-il être tenu sans pour autant recourir au personnalisme?

Procéder à une mise entre parenthèses de sa morale personnaliste et interroger Scheler depuis cette posture impliquent forcément de confronter sa pensée à la tradition phénoménologique ellemême. Les rapports ou les divergences complexes qui permettent de mettre son œuvre en vis-à-vis de celles de ses contemporains allemands, Husserl puis Heidegger, ont été explorés dernièrement (Frère, 2006a $)^{7}$. Il s'agit à présent de s'arrêter sur les avancées de sa phénoménologie que permet de dévoiler la tradition française qui l'a prise en considération. Circonscrire de la sorte l'investigation

\footnotetext{
4. Nous empruntons l'idée d'herméneutique morale schelerienne à l'article de J. Porée (1997, s. 104-116.) répondant à celui de J-L. Lacoste (1997, s. 87-103). Par ailleurs, pour cet article, nous entendrons avec J-Y. Lacoste les termes d'éthique et de morale dans une même acception. "Les faits éthiques sont des faits offerts à une perception matériale, mais assurément pas à une perception sensible. Ce sentir émotionnel est comme tel en situation de connaissance morale" (ibid., pp. 89-90.).

${ }^{5}$. C'est alors dans un personnalisme chrétien que se refermerait son système moral. Sur cette dimension, on consultera M. Dupuy (1959b). Voir aussi entre autres J.P. Chevrolet (1970), L. Lavelle (1936), Y. Ledure (1993), J.-M. Oesterreicher (1955), A.D. Sertillanges (1941).

${ }^{6}$. Nous avons essayé de montrer que l'axe le plus achevé de cette critique est celui par lequel il entend se débarrasser du l'unité synthétique du Je Kantien (B. Frère, "Phénoménologie et personnalisme" in Archives de philosophie, nº67, cahier III, 2004, pp. 445-464).

7. B. Frère, "Scheler critique de Husserl. Esquisse d'une perspective non transcendantale au cœur du projet phénoménologique", in Philosophie, n 91, 2006a, pp. 80-105.
} 
constitue le second objectif de cet article. En soi, il s'agit autant d'une restriction que d'un nouveau pari. En effet, rares sont les commentateurs qui se sont arrêtés sur cette influence, pourtant prégnante, de Scheler sur la configuration française du projet phénoménologique. Pourtant, c'est elle qui peut permettre à la pensée de Scheler de tenir ses promesses.

Seul H. Leroux soulignait qu'il est effectivement possible "de relever, sur d'assez nombreux penseurs français dont la portée est incontestable, des «points d'impacts » de la pensée de Max Scheler : ainsi Mounier, Marcel, Sartre, Merleau-Ponty, Ricoeur, Henry" (1994, s. 332-356). Pour notre part, nous laisserons de côté Mounier et Marcel - qui se sont principalement penchés sur l'aspect personnaliste de l'œuvre de Scheler dont nous désirons précisément faire abstraction - et nous ajouterons à la liste Mikel Dufrenne. Il compte en effet avec Michel Henry, parmi les phénoménologues français à s'être les plus explicitement positionnés par rapport à Scheler. En conséquence, ce sont leurs réflexions qui alimenteront plus particulièrement la nôtre. Mais certains éléments dans les œuvres de Sartre, Ricoeur ou encore Merleau-Ponty peuvent aussi constituer une appui précieux pour répondre à la question de savoir ce que l'intuition affective et l'a priori material sont susceptibles d'apporter à la phénoménologie sans pour autant renvoyer Scheler à ses solutions personnalistes trop rapidement ${ }^{8}$.

L'approche de M. Dufrenne en terme d'apriorité marquera la première étape de cette démarche. Elle permet de poser les bases du débat en déployant l'objectivisme de la pensée de Scheler. Evoluer avec lui de la question matériale à celle de l'essence et du sens, permet de comprendre pourquoi Scheler prend définitivement congé de l'idée de sujet constituant. En rapportant l'intentionnalité à la perception affective au sens strict, nous verrons comment il s'éloigne alors de Husserl (mais pas de la phénoménologie) pour réduire notre relation première aux objets à une visée de «valeurs », c'est-à-dire à l'intuition eidétique de leur essence axiologique. Si a priori il y a, il est à situer du côté de l'objet dans sa valeur, insiste Scheler. Ce qui revient à dire que le pôle subjectif serait toujours déjà second dans la relation intentionnelle qui le lie à l'objet en question.

Après avoir bien saisi la priorité que Scheler octroie à l'a priori matérial, au sens et à la valeur (aux dépens de la conscience ou du sujet), et sans encore faire droit à une herméneutique morale, il deviendra nécessaire de qualifier davantage la nature originairement affective de l'intentionnalité schelerienne. Si tout est effectivement du côté de l'objet, quelle est la modalité de la perception qui m'y rapporte ? Autrement dit, quel est le modèle de mon rapport premier à l'objet ? C'est dans les termes d'une radicale hétéro-affectivité que se déniche alors, au cœur du Formalisme, l'originalité d'une visée qui tracera à la tradition phénoménologique française les bornes d'un débat qui est loin d'être clos. Mais c'est aussi elle qui posera tout l'épineux problème de la fondation de ces valeurs $a$ priori. Ce dernier constituera l'objet d'un ultime paragraphe structuré autour des réflexions que Michel Henry nous laisse à son propos. Entièrement transcendantes, ces valeurs font du moi un être toujours déjà projeté affectivement à l'extérieur de soi à un point tel qu'il devient difficile de le penser en tant que pôle intentionnel. La phénoménologie de Scheler nous amène à nous demander simplement "comment le «je» qui accueille l'apparition d'une valeur nous prouvera-t-il qu'il intervient ici au simple titre d'une conscience intentionnelle" ${ }^{9}$ ?

En conclusion de notre propos la question initiale de cet article se posera à nouveau d'ellemême : La démarche de Scheler, devenue non-husserlienne, ouvre-t-elle des perspectives de recherche insoupçonnées dans le cadre strict de la phénoménologie ? Ou, à l'inverse, les impasses finalement soupçonnées impliquent-elles obligatoirement de faire signe à d'autres schémas de

\footnotetext{
${ }^{8}$. "Aucun d'eux n'avait pour but, quand ils se référaient à Scheler, de tenter une saisie globale de l'œuvre de Scheler pour l'introduire dans la culture française. Leur préoccupation principale fut celle de leur propre chemin. La force de pénétration de la pensée schelerienne n'en est que plus manifeste" écrit Leroux (1994, p. 332.)

9. "à laquelle les choses dictent leur loi" (J.-L Lacoste, 1997, p. 93).
} 
pensée ? Car en fin de compte, s'il nous semble nécessaire de mesurer combien la phénoménologie conditionne à l'origine la morale et l'éthique scheleriennes (plutôt que l'inverse), ce travail ne se suffira pas à lui-même. Il faudra bien aussi se demander si elle peut clore son système comme elle l'a ouvert. Cela reviendra à voir si l'originalité de sa pensée peut inaugurer un futur programme phénoménologique sans recourir aux conclusions personnalistes qui furent les siennes.

\section{§2. Quand l'objet se voit assigner l(')a priorité}

Nous relevions pour commencer que, chez Scheler, le donné matérial ne peut être assimilé aux "constituants sensoriels". Omettre de distinguer l'un et l'autre serait réintégrer la méprise kantienne. La matière qui fait en grande partie l'objet du Formalisme est axiologique. Et "ce qui constitue [...] les «constituants sensoriels» de la matière axiologique (ou ce qui peut être improprement appelé ainsi) n'est jamais donné immédiatement dans cette matière" (M. Scheler, 1955, p. 82). Ils peuvent, au mieux, être donnés a posteriori, comme support matérie ${ }^{10}$. L'affect dont il faut s'occuper est donc un vécu d'un ordre particulier, celui de l'intuition matériale, supérieure à l'ordre non intentionnel des états émotifs sensoriels. Ces derniers ne sont simplement pas pertinents du point de vue phénoménologique car l'affect qu'ils peuvent susciter "ne contient en lui aucune «visée mentale», insiste Scheler. Il n'est aucunement «orienté vers»", contrairement au sentiment dont doit s'occuper le philosophe phénoménologue : celui de la perception affective (p. 269).

Quelle est la raison de ce primat de l'intentionnalité affective sur nos simples émotions sensorielles ? Et qui plus est, quel en est le contenu ? Répondre à ces questions implique de cerner le plus précisément possible le croisement de deux axes fondamentaux dans le Formalisme: celui de l'apriorité matériale et celui du caractère "essentiel" qui lui est assigné. Plus qu'un choix de procédure, c'est une nécessité qu'impose la démarche schelerienne. Car si l'on s'en tient à son usage de la méthode phénoménologique, toute recherche en terme d'intentionnalité doit commencer par "faire abstraction des structures organiques spécifiques des supports d'actes et des positions d'existence effective des objets pour mettre à jour ce qui est à la base même de l'essence de ces sortes d'actes et de leur matière" (p. 87).

Comme le fait très bien remarquer M. Dufrenne, "si l'a priori est donné à une intuition immédiate, ce n'est pas comme une chose, c'est comme une essence" (1959, p. 75). Son mode de donation est l'intuition eidétique. Chez Scheler, poursuit-il, "l'a priori est un fait donné a priori, essentiel et non formel ; car le fait ici, ou le phénomène, c'est l'essence" (p. 78). Tout se passe comme si Dufrenne tentait de penser les notions d'a priori, d'essence et de phénomène chez Scheler dans un même mouvement. Nous ne lui donnerons pas tort, et notre hypothèse recoupera la sienne. Scheler, toujours contre Kant, estime que le phénomène en tant que tel "n'a rien à voir avec la manifestation (d'un réel) ou l'apparence. Il s'agit bien plutôt du «corrélat» d'une vision de l'essence, le quid"de toute chose (M. Scheler, 1955, p. 71). La pure quiddité de l'objet s'identifie encore ici explicitement à un a priori pour lequel "ou bien il est saisi intuitivement et ainsi, donné lui-même [...] ou bien il ne l'est pas et alors il n'est pas donné" ${ }^{11}$. L'a priori (l'essence dont

\footnotetext{
${ }^{10}$. Sur la distinction material- matériel, $C f$ supra note 4.

${ }^{11}$. Scheler se donne donc comme objectif dans le Formalisme le dévoilement du phénomène. "Il est ce qui se donne en personne et non plus un principe de dissimulation" (M. Dupuy, 1959b, p. 248). L'essence se rapporte au phénomène. Or, à partir de Vom Ewigen im Menschen, "le phénomène se confond avec l'être" (ibid.). Scheler se refuse à envisager tout cela dans un rapport à une conscience, l'essence de l'objet (son être, son phénomène) subsistant "indépendamment de l'acte qui la vise et qui la fait apparaître" (ibid.). Ainsi conçue, elle fonde la possibilité de l'ontologie que nous avons déjà étudiée (B. Frère, 2006a). Peut-on donc voir dans cette relation entre l'essence et le phénomène du Formalisme une préfiguration de celle-ci ? Probablement. Dans Phänomenologie und Erkenntnistheorie, Scheler parle plus précisément
} 
s'occupe le Formalisme), ainsi compris, rompt de manière décisive avec son orientation traditionnellement subjectiviste. Il n'a pas à être assimilé à de soi-disant concepts originairement purs au seul moyen desquels l'entendement puisse "comprendre quelque chose dans le divers de l'intuition", tout simplement parce qu'il est lui-même dans l'objet (E. Kant, 1980 [A.80]). Il n'a pas à être le facteur qui "fournit le fondement objectif de la possibilité de l'expérience" ([A. 94]), car à l'inverse, n'est justement, "a priori que ce qui est logiquement indépendant de toute expérience, et non ce qui est antérieur à telle expérience" (M. Dufrenne, 1959, p. 97) ${ }^{12}$. Le coup de force opéré par Scheler est tout sauf négligeable ; l'a priori ne doit plus être conçu "comme condition subjective de l'objectivité mais comme structure privilégiée de l'objet" (p. 103). Je le saisis affectivement et en toute passivité.

Certes, l'a priori constitue l'objet comme objet signifiant à notre égard, mais c'est à "l'état sauvage" qu'il nous faut le considérer écrira plus d'une fois Dufrenne. "Non point tel qu'il apparaît lorsqu'il est élaboré par une réflexion qui l'explicite et l'exploite, mais tel qu'il apparaît immédiatement au cœur de la perception" (p. 71). Scheler le premier l'a compris, et c'est à lui qu'on doit encore les meilleures explications. Mais ce n'est pas tout. Car si Scheler ôte en fait au sujet l'initiative de la constitution (en tant qu'activité subjective unifiant le divers de l'intuition), ce n'est que pour mieux la léguer à l'essence $a$ priori grâce à laquelle l'objet s'annonce lui-même comme objet d'expérience. Voilà pourquoi l'a priori — étant présent dans l'objet comme ce qui le constitue — ne procède plus en aucun cas d'une subjectivité formelle mais d'un strict donné matérial.

Evoquer l'a priori matérial nous éloigne fortement de Kant. On pourrait croire que nous ne sommes pas encore définitivement séparés de Husserl qui contestait également qu'il n'existât d'a priori que formel. La volonté de Scheler est bien celle de mettre un terme définitif au règne que le sujet fait encore, illégitimement, prévaloir sur l'objet. Dans ce contexte il ne refuse effectivement pas qu'un sens s'offre passivement à nous dans l'apparence. Mais il ne peut avoir été subjectivement constitué comme chez Husserl ${ }^{13}$. Si ce sens est saisi c'est dans une extériorité radicale, dans une transcendance matériale irréductible à l'intuition subjective que nous en avons. C'est ainsi que le raisonnement de Scheler rebondit. À peine a-t-il lu l'a priori dans l'essence d'une telle transcendance qu'il accorde que l'a priori n'est plus seulement à ce titre "la structure immanente de l'objectivité mais aussi bel et bien son sens donné dans l'expérience"(M. Dufrenne, 1959, p. 56). L'a priori, envisagé sous la forme d'une essence transcendante répond à l'exigence de la structure signifiante de l'objet. En d'autres termes, il appartient à l'essence d'un objet de se signifier luimême, "et cette signification est a priori d'abord parce qu'elle est immanente à l'objet" (p. 33). Connaître l'objet, c'est ainsi saisir sa manifestation essentiale, appréhender ce qui lui est le plus propre.

de "phénomène phénoménologique" à dévoiler en-deçà du phénomène empirique et "qui se montre et se laisse voir après avoir procédé à la réduction phénoménologique" (R. Wiehl, 1991, p. 179). Ceci fait indéniablement penser à l'approche ontologique d'Être et temps et à la distinction heideggerienne entre le phénomène au sens authentique du terme et le phénomène au sens vulgaire de l'intuition empirique, lequel n'est pas "un se montrer en soi-même mais le fait, pour quelque chose qui ne se montre pas, de l'annoncer par quelque chose qui se montre" (M. Heidegger, 1985, p. 42).

12. L'erreur de Kant dans l'optique schelerienne est définitivement posée en A. 120 : l'auteur de la Critique de la raison pure y écrit que "sans le rapport à une conscience au moins possible, le phénomène ne pourrait jamais devenir un objet de la connaissance et ainsi il ne serait rien pour nous, et puisqu'il n'y a en soi aucune réalité objective, et qu'il n'existe que dans la connaissance, il ne serait absolument rien".

${ }^{13}$. Position que résume le célèbre paragraphe 55 des Idées I: "L'absurdité commence quand on se met à philosopher et que, en quête d'une ultime information sur le sens du monde, on oublie de remarquer que le monde lui-même a son être complet sous la forme d'un certain « sens » qui présuppose la conscience absolue à titre de champ pour la donation de sens; du même coup on oublie que ce champ, ce royaume ontologique des origines absolues (diese Seinssphäre absoluter Ursprünge), est accessible à une investigation intuitive et qu'il se prête à une infinité d'évidences de la plus haute dignité scientifique". Pour le dire vite, toutes les unités réelles sont des « unités de sens » (Einheiten des Sinnes) " et "des unités de sens présupposent une conscience donatrice de sens" (1950, pp. 183-184-185). 
Toujours contre Kant (et contre la possibilité d'une connaissance a priori qui reposerait sur l'entendement) "le savoir de l'a priori n'est donc lui-même en aucun cas un savoir apriorique. Mais il est un savoir qui, bien qu'a posteriori - et sans pour autant être obtenu inductivement — vaut pour tous les objets [...] pour autant qu'ils soient objets d'une telle essence" (M. Scheler, 1954, s. 445, nous traduisons). L'a priori possède donc un sens. Ou plutôt, devrions-nous dire avec Dufrenne, l'a priori est un sens. Il est présent à ce titre dans l'objet, comme ce qui l'habite et le rend susceptible de s'offrir à moi si j'oriente sur lui ma visée - toujours déjà seconde. L'a priori est objectif. Dans un schéma schelerien, on voit rapidement quelle doit être la modalité fondamentale de cette visée tout comme ce qu'elle ne peut et ne doit pas être. Ces courtes analyses nous révèlent un sujet qui, second, se doit d'être "plus ouvert et accueillant que constituant" (1959a, p. 45). L'a priori que Scheler nous invite à considérer est un sens qui ne peut être atteint "par une généralisation et défini rationnellement" (M. Dufrenne, 1959, p. 101). Il n'est pas de la conscience car "il doit être éprouvé sur des objets privilégiés selon l'évidence propre du sentiment". C'est aussi pourquoi il nous faut, à la différence d'un a priori formel, parler avec Scheler de matérialité étant donné que l'a priori n'est rien d'autre dans l'intuition "qu'un contenu qui est immédiatement donné dans l'expérience comme sens de l'objet". L'a priori n'est plus du sujet, mais du monde. Il n'est plus le corrélat d'un savoir intellectuel pur mais du sentiment. Et le sentiment n'est rien moins que "le plus haut moment de la perception, le moment où la perception se fait tout entière perception, où le sujet percevant se voue à son objet, devient en quelque sorte l'objet, du moins le vit jusqu'à s'aliéner en lui" (p. 133).

\section{§3. Le sentiment du sens ou la relégation définitive du sujet}

Il est troublant de remarquer combien les thèses de Dufrenne rejoignent celles du phénoménologue allemand plus encore qu'elles ne le laissent elles-mêmes entendre. Pour lui, il est évident que la reconnaissance de l'objet transcendant doit passer par un acte émotionnel primitif qui consiste "à abandonner le soi et ses états, ses contenus de conscience propres, à les transcender, pour autant que possible, entrer en contact d'expérience avec le monde" (M. Scheler, 1996, p. 64). S'abandonner soi-même "pour avoir part à un autre être comme ens intentionale" est un geste qui conditionne toute connaissance essentielle (p. 63). Or, selon Scheler, on ne comprend plus la vie des émotions comme "ce langage de signes chargés de sens où se manifestent des relations objectives" qui régissent elles-mêmes la totalité de notre propre vie (p. 71). Il nous faut donc opérer un revirement et prendre conscience que "ce n'est ni l'action conquérante ni la pensée dominatrice qui me révèlent le monde comme monde", mais bien le sentiment (P. Ricoeur, 1992, p. 331). Avec le courant qui remonte de Scheler à Dufrenne, montre Ricoeur, l'émotion se manifeste sous la forme "d'une relation au monde plus profonde que celle de la représentation qui constitue la polarité du sujet et de l'objet" (1986, p. 253). La raison logique nous oppose des choses objectivées, représentées, tandis que le sentiment, lui, atteste de notre affinité, de notre harmonie élective avec des réalités dont nous portons l'effigie affective.

Merleau-Ponty le comprendra aussi très bien et peut être lu au plus près de Scheler. L'analyse réflexive, "en voulant survoler l'objet [...] en détruit la structure interne écrit-il. Si je peux rejoindre l'objet, ce n'est pas que je le constitue de l'extérieur, c'est que je m'enfonce dans l'épaisseur du monde par l'expérience perceptive" (M. Merleau-Ponty, 1945, p. 236). L'expérience perceptive à son origine est une affection fondamentale chez Scheler, chez Dufrenne, comme aussi chez Merleau-Ponty ou Ricoeur. Le sentiment relie ce que la connaissance objectivante scinde. Celle-ci tend à m'opposer au monde, alors que le sentiment "unit l'intentionnalité qui me jette hors de moi à l'affection [...]. Ainsi est-il toujours en deçà ou au-delà de la dualité sujet-objet" (P. Ricoeur, 1986, 
p. 264) $)^{14}$. Le logicisme husserlien, pour revenir sur ses prétentions constitutives, court le risque de perdre la transcendance du monde en le considérant comme "immanent à la subjectivité transcendantale" (M. Dufrenne, 1959, p. 111). À cette conception, il faut opposer l'idée que son objectivité ne procède pas d'une objectivation, que l'idée du monde est d'abord, dans son inconditionnalité, sentiment du monde. Celui-ci, disait Scheler dans Le Sens de la souffrance, "entretient et nourrit la soif de l'adhésion à l'être, ainsi que l'enchantement exercé par l'apparence d'indépendance des contenus du monde" (1936, p. 49). Les sentiments perçoivent un sens inhérent à l'expérience même qu'on en fait, impossible à confondre avec leur utilité objectivée.

Force est donc de constater que la critique que ces divers philosophes français adresseront à Husserl, avec une intensité certes variable, recoupe à plus d'un titre le projet de Scheler. Mais, aucun d'entre eux ne se fera non plus entièrement schelerien. Il faudrait par exemple être bien mal inspiré pour ne pas voir que Dufrenne, après avoir reconnu pour un temps ses influences, tentera d'assouplir la notion d'a priori en lui assignant une correspondance du côté du sujet. Le sujet deviendra chez lui celui qui est apte à saisir le sens du monde, ouvert à lui de manière virtuelle et $a$ priori. Son objectif devient alors, au-delà de Scheler, de comprendre l'a priori "comme structure de l'objet et comme savoir au moins virtuel dans le sujet" $\left(1959\right.$, p. 227) ${ }^{15}$. Entre la dette qu'il admet avoir contractée à l'égard de l'a priori schelerien et l'exaltation du sentiment qui l'en rapproche à nouveau, la pensée qu'il développe prendra une orientation bien différente. D'abord, le redoublement de l'a priori qu'il tente d'établir fera immédiatement violence à l'univocité matériale qu'y voyait Scheler. Ensuite, le concept de matérialité lui-même sera chez lui reconduit à la sensibilité (à l'instar de Kant) et ne sera plus en rien justiciable de ce qui chez Scheler faisait figure d'événement autonome : l'intentionnalité axiologique ${ }^{16}$. Ce n'est ni la sensibilité "ni le savoir intellectuel qui met en mouvement notre vivre et notre agir mais la perception affective" continuera à répéter inlassablement Scheler quelle que soit la thématique dont il traite (1953, p. 13.).

Le problème de la préséance de l'a priori matérial se pose ici. En interrogeant l'a priori, le sens que manifeste l'essence de l'objet lui-même dans son autonomie absolue, ne sommes-nous pas tombés dans une auto-constitution objective incompréhensible ? Effectivement, "que ces unités de significations (que sont les valeurs) soient données au sentiment ne ruine nullement leur objectivité" (J-M. Porée, 1997, s. 108). Mais justement, que serait un sens qui ne serait pas relatif à la signification que revêt pour nous l'objet ? Ce problème, Dufrenne semble bien l'avoir compris et c'est peut-être pour cela qu'il nuancera en parlant de sens pour un sujet en ajoutant une correspondance apriorique du côté du pôle percevant. Il faut donc à présent décortiquer la nature même de l'a priori matérial afin de voir ce qui, chez Scheler, permet d'esquiver la menace de cette

\footnotetext{
14; "La raison sans le sentiment reste dans la dualité, dans la distance ; le sentiment nous révèle que, quel que soit l'être, nous en sommes ; Grâce au sentiment l'être n'est pas pour nous le Tout-autre, mais le milieu, l'espace originaire dans lequel nous continuons d'exister..." (p. 260).

15. Selon Dufrenne, Il ne faut pas, comme Scheler, omettre le pôle subjectif de l'intentionnalité car si l'a priori "est promu à l'objectivité et intégré à l'objet, c'est tout de même à l'objet en tant qu'il est connu" (Ibid., p. 140). Le sujet, essentiellement réceptif chez Scheler, est condamné à connaître le sens des choses a posteriori alors que "nous allons à la rencontre des choses, nous anticipons toujours" (Ibid., p. 145). Le sens de l'objet n'est pas par le sujet mais pour lui. "La présence du monde est une présence pour moi" (Ibid., p. 122) et s'il se donne a priori, c'est de manière tout aussi première que la perception qui nous met en présence de lui, "même si nous devons apprendre à percevoir". Comme l'écrit très bien Ricoeur, chez Dufrenne, "de même que l'a priori objectif avait dû descendre de son ciel intelligible dans la pâte du perçu, «de même» il faut faire descendre l'a priori subjectif dans la chair du percevant" (P. Ricoeur, 1992, p. 328). Ce qui entrâne certaines difficultés. "Est-il légitime de casser en deux le problème de l'a priori" demande Ricoeur (p. 333) ? L'en-soi des a priori objectifs et le pour-soi des a priori subjectifs ne sont-ils pas des rendus incommunicables ? Dufrenne relie subjectif et objectif par le sentiment, mais si l'on durcit au départ la dualité, "si on la tient pour réelle, il n’y a pas de raison pour que l'affinité révélée par le sentiment ait jamais un sens".

${ }^{16}$. On doit voir ici une des raisons pour lesquelles, alors qu'il tenait à sa disposition et qu'il utilisait la traduction de de Gandillac dont l'autorité est généralement reconnue, Dufrenne continue à utiliser le terme matériel et non le néologisme matérial qui se répandait alors dans les milieux scheleriens français.
} 
objection. Atteignant progressivement le nerf central du Formalisme, c'est alors lui qui va nous permettre de comprendre ce sens «essentiel» comme ce qui précède en réalité toute visée : la hiérarchie des valeurs. La relation qu'entretient l'a priori à l'objet correspond dans le Formalisme à celle que la valeur entretient avec ce que Scheler appelle un bien ou une chose de valeur. Les valeurs se rattachent aux choses dans lesquelles elles viennent apparaître et pour l'expérience desquelles elles sont a priori. Elles "ne sont pas des choses (Dinge), mais des choses nous apparaissent comme porteuses de valeurs" (1997, s. 89)

La hiérarchie axiologique est supportée par le monde de notre attitude naturelle. Si une valeur apparaît à l'affect, c'est sur l'objet (dans lequel Scheler entend aussi la chose de valeur) qu'elle habite. "C'est en lui que la valeur est à la fois objective et réelle" (Dufrenne, 1959, p. 106). Voilà le rapprochement qui permet, dans le sillage de Dufrenne, de montrer comment Scheler comprend le phénomène constitutif indépendamment de tout sujet constituant. "La valeur s'annonce dans le bien parce que le bien est constitué par la valeur. Dès lors, si les valeurs se donnent comme $a$ priori $-a$ priori pour les objets qu'elles constituent, et cela suffit [...] à garantir leur apriorité — elles se donnent sur les objets" comme le sens de ces objets (p. 107). Bref, dans la valeur se trouve le dénouement du problème de la nature strictement objective du sens et de l'a priori.

Scheler ramène aux objets (choses de valeurs) dans leur plénitude axiologique l'a priori objectif dont je perçois affectivement le sens dans mon "échange sentant et vivant avec le monde" (1955, p. 90). Sur un objet pratique, dit le Formalisme, repose "un objet axiologique en général [...]. Ce qui constitue ces objets ce ne sont pas les choses de la perception ou de la représentation, mais les choses de valeurs" (p. 152) ${ }^{18}$. Ce n'est d'ailleurs que postérieurement à notre relation originaire à des unités de sens et de valeurs que ces choses vont pouvoir devenir "absolument parlant des objets pratiques". C'est dire que ce n'est qu'à la suite d'une intentionnalité axiologique — qui est dans sa nature affect, sentiment - que nous pouvons envisager de porter un jugement rationnel et objectivant sur les choses qui les portent. Avancer que l'objet et le rapport que nous entretenons avec lui sont déterminés par sa valeur ou encore par "sa vérité objectale", revient, somme toute, à poser une condition absolue : "ce n'est qu'autant qu'on a découvert dans les res elles-mêmes les constituants essentials aprioriques [...] que tous les principes et tous les concepts de l'entendement trouvent la plénitude de leur contenu" (p. 97). Bien avant elles, dans l'apriorité de ces constituants essentiels de chaque chose "se révèlent les constituants existentiels et axiologiques" du monde des valeurs. Lesquels ne sont pas davantage saisissables par un acte purement intellectif que par la seule sensibilité. Scheler renverse en quelque sorte le rapport constitutif pour s'assurer qu'aucune initiative ne puisse être envisagée du côté du sujet percevant.

La valeur matériale d'une chose se "signifie" seule à nos yeux en tant "qu'unité axiologique phénoménalement donnée" (p. 162) ${ }^{19}$. Elle n'est pas ajoutée ou adjointe du dehors, mais elle se lit

\footnotetext{
17. "Les valeurs existent réellement, matérialement, sur le mode original de ce qui n'apparaît qu'à l'affect" (p. 91). Valeur, essence, sens, autant de synonymes qui, portés par l'objet, constituent l'a priori objectif perçu par l'intentionnalité première, c'est-à-dire par l'affectivité.

${ }^{18}$. Ici, Scheler s'en prend à la "perception" au sens classique du terme, cette perception n'a rien à voir avec ce qu'il nomme "la perception affective" (cf. infra).

${ }^{19}$. Scheler donne un exemple pour bien faire comprendre toute la spécificité de l'intuition matériale axiologique. Si je me retrouve face à un paysage ou à un tableau, ma première réaction pourrait être d'exprimer un «ah !» traduisant mon étonnement, une sensation agréable ou n'importe quel autre sentiment sensiblement provoqué. "Le «ah !» ne vise ni ne signifie rien. Il se contente d'exprimer un état affectif" (p. 190). Par contre, "les phrases «ce tableau est beau», «ce paysage est aimable» visent et signifient quelque chose, et quelque chose qui se trouve dans le tableau, dans le paysage". L'état affectif, ne possède pas le caractère intentionnel qui appréhende le beau et l'aimable dans ces choses. Par contre, il existe dans le sentiment de ces valeurs une intentionnalité pré-logique qui se concrétise par "la saisie des matières axiologiques présentes dans les objets". La valeur, dans la perception affective, peut alors continuer à nous être donnée "sans que ne nous soit donné le support de cette valeur" (p. 41). La réalité ou la non-réalité de la valeur n'a plus la moindre importance, le tableau ou le paysage peuvent disparaître, leurs valeurs seront toujours percevables en tant que
} 
sur elle dans une immédiate passivité affectivo-percevante. Finalement, Kant n'avait pas tort de soutenir que l'a priori était une loi universelle "sous laquelle seules des choses peuvent devenir objets de notre connaissance en général"(1985b, [V. 182]). Mais au-delà de cette commune prémisse, on sait combien Scheler reprochera à Kant de s'être fourvoyé dans l'assignation de cette loi en maintenant que c'est l'entendement qui "pour la faculté de connaître, contient les principes constitutifs a priori"([V. 196]). Scheler nie catégoriquement que notre attitude première face au monde puisse être ce qui lie un entendement à son objet. Ce pourquoi elle doit être entièrement révisée. Elle n'est ni exclusivement ni primitivement "une attitude «représentative», une attitude de perception" au sens entendu par Kant, mais bien "plutôt et d'abord une attitude de saisie axiologique et de perception-affective des valeurs" (1955, pp. 213-214). Ainsi, l'objet de l'attitude naturelle auquel, selon Scheler, l'intellectualisme husserlien restera rivé, trouverait son fondement dans sa propre unité axiologique.

\section{§4. De la transcendance du sentiment à l'a priori axiologique : Intentionnalité et hétéro-affection}

À première vue, l'évolution de Scheler s'avérerait sans faille. De l'a priori axiologique et matérial à son caractère objectif et essentiel, de l'essence à la préséance du monde et de ses res (objets, biens, choses de valeurs), la conséquence serait donc bonne. Il demeurerait fídèle à sa logique radicalement anti-subjectiviste. Mais une difficulté de taille empêche de refermer là le dossier ouvert sur sa phénoménologie. A force de manifester continuellement la volonté "de donner la parole aux choses plutôt qu'aux théories", de subordonner le sujet au monde axiologique et matérial vers lequel il ne lui reste plus qu'à se transcender, l'œuvre de Scheler deviendra sujette à caution aux yeux de certains (M. Dupuy, 1959a, p. 715) ${ }^{20}$.

La critique sévère que fait $M$. Henry de l'intentionnalité affective en général et de la conception de Scheler en particulier illustre très bien les réserves qui peuvent être émises à l'égard de son objectivisme. L'auteur de L'Essence de la manifestation reconnaît à Scheler d'avoir enfin élevé l'affectivité au statut qui lui revenait de droit. Il saluera en ce sens les efforts déployés pour l'arracher aux sphères de la perception sensible, de l'intuition intellectuelle ou de l'entendement qui se l'étaient accaparé jusqu'alors. On peut lire sous sa plume qu'avec Scheler, l'affectivité, "dans son autonomie souveraine, se laisse comprendre pour ce qu'elle est, [...] un pouvoir de révélation originaire propre" (1963, p. 716). Son projet est louable dans son ambition : montrer que ce pouvoir de révélation conditionne la représentation ou, pour être plus précis, "se révèle antérieurement à l'objet de la représentation et se comporte chaque fois comme un guide pour la détermination de ce dernier" (p. 718). Mais très vite, estime Henry, le penseur allemand se trompera sur le véritable enjeu de cette révélation, en perdant l'affectivité dans la transcendance et l'affecté dans l'intentionnalité.

D'après Scheler "dès l'origine, la perception affective comporte «une relation de soi» et une orientation de soi vers un objectal, vers des valeurs" (1955, p. 270). Avec le sentiment, nous sommes

\footnotetext{
matière d'une affection. Merleau-Ponty reste proche de Scheler. Il écrit pareillement qu'en lui-même, "le monde n'est pas donné, et rien n'en est accessible à ceux à qui les sensations sont données. Le perçu n'est pas nécessairement un objet présent devant moi [...] il peut être une valeur" (1945, p. 370-371). Et comme pour prolonger l'exemple donné par Scheler, il émet que "si l'on a enlevé un tableau d'une pièce où nous habitons, nous pouvons percevoir un changement sans savoir lequel" (p. 371). Comme si l'intuition d'une valeur persistait, mais que plus rien ne pouvait la remplir.

20. "Ainsi la perception nous sera présentée avant tout comme une évasion du sujet hors de sa sphère et comme une pénétration dans un domaine étranger où la conscience atteint des déterminations dont elle n'est pas l'auteur et qui, au moment même où elle les connaît, continuent de lui paraître et de lui être transcendantes" (ibid., p. 398).
} 
en présence d'un "mouvement dans lequel quelque chose m'est donné et «se manifeste à moi»". En distinguant les simples états affectifs sensoriels de la perception affective elle-même, il rejoint selon toute vraisemblance la différence que Husserl établit entre l'intentionnel et le non-intentionnel ${ }^{21}$. Aussi, "pour les vécus que nous désignons en général sous le nom de sentiment, il est absolument indiscutable qu'ils ont une relation intentionnelle à quelque chose comme un objet" (Husserl, 1961, $\S 14$, p. 192). Quoique Husserl n'envisage pas, comme Scheler, qu'il puisse exister des sentiments exempts de tout rapport à la sensibilité, ce dernier devra lui concéder la juste mise en valeur de la transcendance de l'objet de l'intentionnalité affective. Pour l'un et l'autre, il faudra s'affranchir des thèses selon lesquelles "le sentiment considéré en lui-même ne renferme rien qui ne soit intention" et donc "ne renvoie pas, au-delà de lui-même, à un objet ressenti". De cette interprétation du sentiment, certains tireront les conséquences qui s'imposent et estimeront que ce n'est alors que "par son unification avec une représentation qu'il acquiert une certaine relation à l'objet" (p. 193). Conclusion des plus inexactes, puisque c'est dès leur apparition que nos vécus émotionnels doivent être compris en leur essence comme étant relation intentionnelle. Émotion portée sur...

Sartre explorera le terrain de l'intentionnalité émotive préalablement dégagé par Husserl puis par Scheler (ce faisant, ses théories en la matière devront d'ailleurs également tomber sous le joug de la critique henryenne). Non sans faire signe aux deux philosophes, il insistera à plusieurs reprises sur la manière dont les sentiments "représentent une façon parmi d'autres de se transcender" (1940, p. 137). Ils donnent bien "une espèce de connaissance". En d'autres mots, le sentiment vise un objet mais il le vise à sa manière, qui est affective. Pour Sartre comme pour Scheler, il s'agit explicitement d'éviter, dans l'affectivité, les risques de tout solipsisme, de toute tonalité individuelle pure, qui demeurerait "enfermée dans le sujet qui l'éprouve" à un stade où le monde des choses n'existerait pas encore (p. 136) ${ }^{22}$. Aussi sommes-nous conviés à nous écarter d'une émotion qui "s'éloignerait de l'objet pour s'absorber elle-même" pour nous rapprocher de celle qui "revient à chaque instant sur l'objet et s'y alimente" (J-P. Sartre,[1939], 1995, p. 39).

En prenant le risque de parier contre l'intentionnalité en général, et donc, dans le cas qui nous occupe, contre l'intentionnalité émotionnelle en particulier, M. Henry prendra donc le contre-pied de Husserl, certes, mais aussi celui de Scheler et de Sartre. Comme on le sait, Henry estime qu'en situant la structure fondatrice $\mathrm{du}$ pouvoir de l'affectivité dans la transcendance, ces phénoménologues ont totalement ignoré l'essence de ce pouvoir lui-même. Entendons qu'en fait, ils se sont gardés à tort de poser la question décisive "du fondement du caractère affectif de la perception affective" (M. Henry, 1963, p. 734). Cette ignorance de la question décisive a permis d'identifier le pouvoir de révélation de l'affectivité à l'intentionnalité et d'alléguer le primat de son contenu : l'objet transcendant. Pour la philosophie occidentale dit Henry, en faisant toujours allusion à la visée intentionnelle, "ce qui ne porte pas en soi cette structure et ainsi ne se rapporte à rien, ne se transcende vers aucun objet, est par principe dépourvu d'un tel pouvoir, du pouvoir d'accomplir une révélation quelconque" (p. 724). Sans transcendance, pas de manifestation et à l'inverse, pas de transcendance sans manifestation pourrait-on dire. Or, pour Henry, c'est là ne pas voir la véritable nature du sentiment qui se constitue justement par l'exclusion de toute transcendance et ne se révèle

\footnotetext{
${ }^{21}$. Dans L'Être et le Néant, Sartre traite de la particularité de l'affection prise comme intention transcendante. Scheler, convient-il, "a bien marqué que cette «intention» doit se distinguer des qualités affectives pures. Par exemple, si j'ai «mal à la tête», je puis découvrir en moi une affectivité intentionnelle dirigée vers ma douleur pour la «souffrir», pour l'accepter avec résignation ou pour la rejeter, pour la valoriser (comme injuste, comme méritée, comme purifiante, comme humiliante, etc.), pour la fuir. Ici, c'est l'intention même qui est affection, elle est acte pur et déjà projet, pure conscience de quelque chose" (1943, p. 370).

22. "Si j'aime les longues mains blanches et fines de telle personne, cet amour, qui se dirige sur ces mains, peut-être considéré comme une des façons qu'elles ont d'apparaître à ma conscience. C'est bien un sentiment qui vise leur finesse, leur blancheur, la vivacité de leur mouvement : que signifierait un amour qui ne serait pas amour de ces qualités ? C'est donc une certaine façon de m'apparaître qu'ont finesse, blancheur et vivacité". Mais, comme aurrait pu le dire Scheler, "ce n'est pas une connaissance intellectuelle" (p. 138).
} 
qu'à soi-même.

Il y va ici de sa conception de la tonalité que Scheler ou Sartre refuseraient de front. Henry estime que la tonalité de chaque sentiment est une auto-révélation particulière et immédiate qui s'enracine, en son essence, dans "l'immanence radicale" de l'auto-affection (p. 771). Ce que présuppose la représentation, ce n'est pas une "donnée hylétique de l'impression pure" ${ }^{23}$ ou le sentiment du contact avec une chose de valeur, bref en tous les cas, un objet transcendant et une hétéro-affectivité, mais bien "l'affectivité du sentiment lui-même, son être originaire et propre" (p. 716). Parler d'un tel objet de sentiment (par exemple en termes de valeurs), en faire un corrélat noématique, c'est déjà plonger dans la représentation. Car, demande-t-il, que serait un sentiment qui ne s'éprouve pas soi-même intérieurement et ne se donne pas originellement à lui-même dans son affectivité sinon, précisément, déjà une représentation ? La perception de l'objet qui m'affecte (Scheler) ou sa représentation (Husserl) «valent pour» le sentiment mais ne rendent pas comme telle la manière dont il surgit dans le «s'éprouver soi-même intérieurement » de l'auto-affection. "Le fond du sentiment se résorbe dans l'incapacité de s'exhiber, d'une manière générale, dans le milieu de l'être transcendant" (p. 818). Il précède de la sorte la reconnaissance de l'objet à l'origine d'une affection, parce que "c'est partout et toujours de toute tonalité quelle qu'elle soit, que doit être affirmé son auto-surgissement, à partir de soi comme déterminant ce qui l'affecte, comme identique à la réalité de cette affection" (p. 616).

\section{§5. Conclusion. L'intentionnalité affective : impasse ou nouvelle piste phénoménologique?}

Avoir ainsi sommairement convoqué l'originalité d'une phénoménologie qui prétend faire fi de l'intentionnalité avait pour objectif "d'aménager le terrain" sur lequel surgit la spécificité de la critique que Henry adresse à Scheler. Ce débat à quatre voix (Husserl, Henry, Sartre, Scheler) nous permet de la reprendre dans le prolongement des concepts clefs déjà précisés : sentiment, matérialité, apriorité, intentionnalité affective et axiologique. Comment l'articulation de ces concepts est-elle susceptible d'être bousculée par une philosophie qui affirme "que l'affectivité est irréductible à la relation intentionnelle comme à toute forme de transcendance en général" (M. Henry, 1963, p. 816)?

Nous avons vu que la transcendance chez Scheler n'est plus celle des choses saisies par des actes objectivants - comme pour Husserl - mais correspond aux "éléments constituants de nos entours [...] phénoménalement revêtus de valeurs", saisis par perception affective (1955, p. 256). Ce qui reste toujours donné dans la transcendance, "c'est la place des valeurs appartenant aux objets, c'est, absolument parlant, l'être axiologique objectal" disait-il. En toute logique, on trouve là ce contre quoi se dressera Michel Henry : l'a priori matérial dans son acception axiologique ${ }^{24}$.

\footnotetext{
${ }^{23}$. D. Giovannangeli, 1995, p. 23. M. Henry parle bien, à l'instar des Leçons pour une phénoménologie de la conscience intime du temps (1964), d'impressionnalité (le pathos de l'origine). Ce qui ne veut pas dire que chez Husserl, celle-ci répondait par anticipation à l'exigence henryenne, bien qu'Henry lui-même reconnaisse là le texte "le plus beau de la philosophie de ce siècle" (M. Henry, 1990, p. 31).

24. La mise à l'épreuve du système schelerien deviendrait d'ailleurs d'autant plus sévère que nous pourrions lui adjoindre la conclusion de l'Être et le Néant. Ici, le blâme que Sartre adresse à "l'esprit de sérieux" semble bien être résolument orienté contre lui. L'esprit de sérieux a pour double caractéristique "de considérer les valeurs comme des données transcendantes indépendantes de la subjectivité humaine, et de transférer le caractère «par exemple désirable» de la structure ontologique des choses à leur simple constitution matérielle" (1943, p. 674). Dans cette perspective, et c'est bien là, selon nous, l'optique de Scheler, "les objets sont des exigences muettes, et il n'est rien en soi que l'obéissance passive à ces exigences" (1955, p. 256.). Plus que la hiérarchie axiologique elle-même, ce sera souvent la
} 
L'intuition géniale qui fut celle de Scheler est d'avoir repéré "que la découverte des éléments qui conditionnent notre compréhension du monde et qui la guident originellement, ne s'accomplit pas sans la présence de sentiments déterminés" répète M. Henry (1963, p. 734). Il a très judicieusement détaché les sentiments de la stricte sensation. Mais pourquoi avoir fait de ces éléments des valeurs matériales ? Il n'est pas contestable que Scheler a bien fait de ne pas suivre Husserl dans son attitude objectivante. Mais le résultat auquel il aboutit en définitive n'est pas différent. Chez lui, ce sont des valeurs qui se présentent, "sous la forme d'un corrélat intentionnel, comme d'un contenu extérieur", lequel s'enracine "dans la structure ontologique de l'objet et lui est identique"(M. Henry, 1963, p. 722).

C'est alors à la lumière de cette signification ontologique structurelle de la transcendance que doit se comprendre l'appartenance au monde de qualités affectives. Nous l'avions relevé avec Dufrenne, la perception affective "lit" pour ainsi dire la valeur a priori sur son support (le bien ou la chose de valeur). Il est donc troublant de constater qu'ultimement, ce sur quoi porte le soupçon de la critique henryenne est précisément cela même que Dufrenne avait fait valoir comme une avancée : le sens objectif et a priori d'une chose non constituée subjectivement. À cet égard continue Henry, Scheler en arrive à se méprendre complètement sur la nature du sentiment qui serait alors de supporter "une signification extérieure, une signification transcendante". L'erreur de la transcendance se confondrait alors avec celle de l'axiologie, la matière de l'intentionnalité affective n'étant rien d'autre que la valeur. En omettant d'envisager l'origine de toute tonalité affective particulière dans l'unité d'une auto-affection, il perdrait les sentiments dans leur propre "extériorité" (M. Scheler, 1955, p. 347). Il serait, par exemple, de l'essence de la visée amoureuse de se rapporter à un objet (une chose de valeur) qui se "signifie" comme étant aimable ou de celle du désir de se rapporter à un objet qui se signifie comme "désirable" quel que soit celui qui vise. Henry conclut en conséquence que Scheler en est arrivé à discuter l'affectivité dans une totale "obscurité ontologique" étant donné que l'on ne sait pas où elle se manifeste (1963, p. 778). Lui situera cette manifestation dans l'auto-affection.

Sans pour autant opter sans partage pour la thèse de l'auto-affectivité, il faut bien reconnaître que la pertinence de la lecture que fait Henry peut laisser perplexe. Scheler propose une conception de l'intentionnalité originale, axée sur la passivité (ce qui le rapproche de Husserl) et l'affectivité (ce qui l'en éloigne). Henry se verra accusé de faire une entorse grave à la méthode phénoménologique en allant jusqu'à remettre en question l'intentionnalité elle-même ${ }^{25}$. Rien de tel chez Scheler. Mais existe-t-il chez lui une unité dans l'affectivité ? En quoi des sentiments différents et hétérogènes sont-ils autorisés à revêtir le nom unique de perception affective ? Est-il seulement un lien possible entre les différentes modalités du sentiment étant donné que chacune d'entre-elles porte sur des objets de valeur, de sens différents ? Qui perçoit tel objet comme nécessairement, objectivement et aprioriquement aimable et tel autre comme nécessairement, objectivement et aprioriquement désirable?

Nous avons voulu attester de la dimension rigoureusement phénoménologique de l'œuvre de Scheler dans cet article. A son issue, nous devons reconnaître d'ultimes problèmes auxquels ni la critique de Kant ni celle de Husserl ne peut encore permettre de répondre. Deux d'entre eux apparaissent majeurs. Le premier pourrait bien être celui du lien entre mes affections. Dans une perspective henryenne, nous pourrions rapporter l'unité des perceptions des valeurs à l'autoaffection. Mais chez Scheler ? Comment nommer la cohésion de mes sentiments portant sur l'essence des choses, à savoir sur leur valeur ? Ni Cogito, ni Je transcendantal, ni conscience, ni

passivité dans laquelle nous devons nous situer à son égard que l'on reprochera à Scheler (cf. par exemple, entre beaucoup d'autres, l'article de M. Loreau (1957, n²0, p. 1-24).

${ }^{25}$. On consultera notamment sur cette remise en question de l'identité phénoménologique de la pensée henryenne R. Bernet (1994, pp. 297-327), D. Janicaud (1990, pp. 57-73), F-D. Sebbah (2001, pp. 93-108), S. Laoureux (2004a), B. Leclercq (2004). 
sujet, c'est bien la personne qui est convoquée comme par nécessité. C'est en fin de compte elle qui lie ses propres actes et qui se trouve être "l'unité immédiatement vécue" de mes différentes perceptions affectives (1955, p. 377. Trad. mod.). La personne vit dans chacun de ses actes, mais elle est aussi capable "de se reprendre, de se ressaisir en vue de se ramasser sur un centre, de s'unifier" (E. Mounier, 1949, p. 87). Ainsi l'unité et l'intériorité reprennent sens, parce que "je fais le bilan des fidélités qui me rassemblent et me confèrent, comme par surcrôt, une identité" (P. Ricoeur, 1992, p. 201).

Certes, Scheler, à sa décharge, aboutit au personnalisme par une voie originale qui n'est ni celle d'une métaphysique chrétienne ni celle d'une philosophie morale mais celle de la toute jeune phénoménologie. Mais cela valait-il bien la peine de procéder à une aussi riche déconstruction du sujet pour en arriver à résorber l'initiative donnée successivement à l'a priori matérial, à l'essence et à la valeur dans l'unité de la personne ? En fin de compte, Scheler tente de critiquer Husserl avec ses propres armes et sur son propre terrain phénoménologique mais il finit par proposer une alternative qui ne peut se déployer que dans une sphère teintée de sacré. C'est en faisant de la personne plutôt que du sujet le nœud de la phénoménologie que Scheler entend dépasser Husserl. Si ses critiques portant sur lui ou sur Kant sont, comme nous l'avons vu, souvent le lieu d'un déploiement original de la phénoménologie et celui d'innovations conceptuelles importantes, Scheler glisse ultimement vers un personnalisme moins original et, à l'époque, déjà fort balisé. Dufrenne l'a probablement compris et ré-intégrera le sujet dans le débat sur l'a priori.

Le second problème que nous évoquerons pour finir est lié de près au premier puisqu'il pose la question du cadre dans lequel évolue le personnalisme schelerien. Les dédales de sa pensée conduisent à voir en l'essence de chaque chose ce qui en fait sa valeur propre, a priori et matériale. Quelle est alors l'origine des valeurs étant donné qu'elles me sont originairement transcendantes, et que le principe de synthèse constitutive est définitivement éludé ? Etant donné qu'il a rejeté toute activité subjective, on comprend qu'une forme quelconque d'intersubjectivité ou de socialité transcendantale (à la façon de la cinquième Méditation cartésienne de Husserl) ne puisse pas avoir l'initiative de la "création des valeurs"26.

La réponse se laisse forcément deviner. C'est ici un Dieu originairement constituant qu'il faut accepter. Il crée le monde des valeurs que nous pouvons saisir sur leurs supports dans une stricte passivité par émotion, intuition affective et axiologique. Chaque perception affective saisissant l'être d'un objet "témoigne seulement de l'infinie richesse d'un monde dont l'accès n'est possible que par un abandon total de soi " (M. Scheler, 1951, p. 136). On relève encore combien les questions que Scheler est amené à poser dans ses développements phénoménologiques trouvent leur réponse ailleurs, dans ce qui, surtout pour le Formalisme, s'esquisse sous la forme d'une métaphysique chrétienne. Toutes les valeurs se groupent alors pour lui "sous l'appel singulier d'une personne suprême" (E. Mounier, 1949, p. 80). Ainsi, chaque personne individuelle ne cessera "de se rapporter à un ciel fixe de valeurs" qu'elle ne constitue cependant pas et vis-à-vis duquel elle devra se résoudre à la plus stricte passivité (P. Ricoeur, 1992, p. 195).

Le commentaire de Landsberg, bien qu'il lise Scheler de manière peut-être encore plus religieuse que Scheler ne se serait lu lui-même, cerne bien toutes les conséquences de ces glissements et nous indique les risques qu'une phénoménologie schelerienne encourt lors de cette transition sans concession du sujet à la personne. Que ce soit dans sa période catholique ou dans son ultime panthéisme (dit pan-enthéisme ${ }^{27}$ ), à force de vouloir pénétrer par le sentiment la richesse inépuisable du monde, Scheler ne risque-t-il pas "de se perdre dans la plénitude de ses rencontres"

\footnotetext{
${ }^{26}$. On trouve ici toute la critique de M. Loreau qui reproche à Scheler de ne pas avoir vu qu'il existe une conscience constituante des valeurs qu'il faut envisager dans "ses rapports concrets avec d'autres consciences" $\left(1957, n^{\circ} 24\right.$, p. 23$)$.

27. Voir H. Leonardy (1981). Sur le pan-enthéisme voir surtout Ch. Krause (1828).
} 
(1952, p. 171) ? À force de secondariser le sujet, l'entendement ou la raison, Scheler n'abolit-il pas toute conscience de soi ? La contradiction semble insoluble... À moins d'envisager sa pensée sous un angle qui réclamerait un travail d'une autre ampleur au sein duquel il en irait de deux choses l'une. Soit on poserait a priori que la recherche engagée est d'ordre éthique, religieux et métaphysique, ce qui implique de laisser de côté la démarche exclusivement phénoménologique que nous avons essayé de tenir ici. Soit on poserait, a posteriori, et à la suite de ces quelques pages, que l'hypothèse d'un tournant théologique de la phénoménologie est judicieuse. Mais ce dernier ne doit alors être situé ni dans la phénoménologie française, ni aussi tardivement que ce que pense D. Janicaud (1990). Mais parallèlement à la naissance du système husserlien, ... avec la philosophie de M. Scheler.

Notre question introductive trouve sa réponse. Oui, Scheler perd bien une partie de l'aspect novateur de sa phénoménologie en hybridant celle-ci avec des courants philosophiques déjà bien fréquentés (ici le personnalisme, là bas le panthéisme ...). Sa pensée ne peut rester homogène sans faire appel à un «dehors » de la phénoménologie. Néanmoins l'originalité avec laquelle il use de cette méthode vaut que l'on s'y attarde. C'est probablement en poursuivant sur la réception francophone de ses textes (et peut-être plus particulièrement à partir des travaux de Dufrenne et Henry) qu'une phénoménologie du sentiment peut encore trouver à s'affiner. Dans l'état actuel des choses, celle-ci s'est déjà avérée très utile au-delà du strict travail philosophique ( $\mathrm{S}$. Laoureux, 2004b, L. Boltanski, 2004, B. Frère, 2006b, 2006c). Il reste à espérer que la tradition phénoménologique francophone continue de se pencher sur celui qui en fut le principal artisan. 